\title{
HELIOSEISMIC CONSTRAINTS ON THE SOLAR STRUCTURE
}

\author{
Evidences of Element Segregation and Mild Mixing below the Convection Zone
}

\author{
S. VAUCLAIR \\ Laboratoire d'Astrophysique de Toulouse \\ 14, avenue Edouard-Belin, 31400-Toulouse, France
}

\section{Introduction}

The study of the internal structure of the Sun entered a new age with helioseismology. Several ground based networks, as well as the space mission SoHO, continuously observe the solar oscillations. In particular GONG, the "Global Oscillation Network Project", gathers six sites around the world with six identical Doppler instruments. These instruments observe the phase shift of the $\mathrm{Ni} 676.8 \mathrm{~nm}$ line with 3 images every minute, 1.8 sites observing simultaneously. Millions of solar p-modes have been detected. The inversion of the measured frequencies yields accurate and detailed information about the sound velocity in the Sun's interior, which in turn leads to constraints on the equation of state, opacities, chemical composition. Precise informations on the differential rotation inside the Sun have also been obtained. (See the special Science issue on GONG, vol 272, 31 May 1996).

The Sun is the best known of all the stars. Its mass, radius, luminosity and age are known with a high degree of precision (see values and references in Richard et al. 1996, herafter RVCD). The photospheric abundances have also been precisely determined (Grevesse 1991). For the light elements, the abundance determinations show that lithium has been depleted by a factor of about 140 compared to the protosolar value while beryllium is generally believed to be depleted by a factor 2 . These values have widely been used to constrain the solar models (e.g. RVCD). However, while the lithium depletion factor seems well established, the beryllium value is still subject to caution. Balachandran (1997) argues that the beryllium depletion is not real because of insufficient inclusion of continuous opacity in the abundance determination. Her new treatment leads to a solar value identical to the meteoritic value.

Observations of the ${ }^{3} \mathrm{He} /{ }^{4} \mathrm{He}$ ratio in the solar wind and in the lunar rocks (Geiss 1993, Gloecker and Geiss 1997) show that this ratio may not have increased by more than $\cong 10 \%$ during these last $3 \mathrm{Gyr}$ in the Sun. While the occurence of some mild mixing below the solar convection zone is needed to explain the lithium depletion and, as we will see below, is consistent with helioseismology, the ${ }^{3} \mathrm{He} /{ }^{4} \mathrm{He}$ observations put a strict constraint on its efficiency.

In the present paper we discuss the constraints on the element segregation and mixing processes occuring in the Sun, obtained from helioseismology and abundance measurements. 


\section{Element segregation induced by microscopic diffusion in stars}

Element diffusion inside the stars represents a basic physical process which cannot be ignored in the computations of stellar structure. When stars form out of gas clouds, they built density, pressure and temperature gradients which force the various chemical species present in the stellar gas to move with respect to one another. As a consequence, the abundances observed at the surface of stars are not always representative of their protosolar values, even if they did not suffer any nuclear processing.

The resulting abundance variations depend on several effects. What we use to call "microscopic" diffusion of the chemical elements represents in fact a competition between two kinds of processes. First the atoms move under the influence of external forces (due to gravity, radiation, etc.), second they collide with other atoms and share the acquired momentum with them in a random way, which slows down their motion. This competition leads to a process of element segregation with a time scale decreasing with increasing density.

Although recognized by the pioneers of the study of stellar structure, this fundamental physical process was long forgotten in the computations of stellar models, except for white dwarfs (Schatzman, 1945). Only with the discovery of large abundance anomalies in main-sequence type stars (the so-called Ap and Am stars), which present characteristic variations with the effective temperature, was element diffusion brought into light fifty years later (Michaud, 1970, see other references in Vauclair and Vauclair, 1982).

At that time, the effects of element diffusion were supposed to be important only when the diffusion time scale was smaller than the stellar age. In the Vauclair and Vauclair (1982) review paper, Fig. 1 shows the regions in the HR diagram where diffusion could lead to "observable" abundance variations. The Sun was excluded, as diffusion could not lead in it to abundance variations larger than some ten percent. At that time evidence of abundance variations could be obtained through spectroscopic observations only, and there was no hope to be able to detect differences of order ten percent.

In the present days, due to helioseismology, we know the internal structure of the Sun with a high precision, and evidence for the occurence of element diffusion is obtained with a high degree of accuracy. Abundance variations of the order of a few percent now become indirectly detectable, by comparisons of the theoretical computations with the results of the inversion of pulsating modes. The confirmation by helioseismology of the predictions concerning element diffusion in the Sun represents a great success for the theory of stellar structure.

\section{Evidence of Element segregation and mixing in the Sun from helioseismology}

Many authors have computed the gravitational and thermal diffusion of helium and heavier elements in the Sun with various approximations (Cox, Guzik and Kidman, 1989; Bahcall and Pinsonneault, 1992; Proffitt, 1994; Thoul, Bahcall and Loeb, 1994; Christensen-Dalsgaard, Proffitt and Thompson 1993; Richard et al., 1996; etc.). Most models, obtained with various codes and numerical techniques, are consistent with each other, which is quite encouraging.

In RVCD, computations of the solar internal structure have been done with the Toulouse code, a version of the Geneva stellar evolution code in which we have precisely included microscopic diffusion processes and possibilities of testing the effects 
of macroscopic motions like turbulence and stellar winds. Microscopic diffusion was treated as in Charbonnel, Vauclair and Zahn (1992), and the diffusion coefficients were computed using the Paquette et al. (1986) approximation. The abundance variations were followed separately for helium and 14 heavy elements, and they were iterated so that the final abundances correspond to those given by Grevesse (1991).

These models have been compared to the results of helioseismology in collaboration with the Warsaw group (Dziembowski et al., 1994). The values of the function $u=P / \rho$ as obtained from the models are compared to those of the "seismic Sun" (Figure 1). The best solar model is obtained by taking into account both the element segregation induced by diffusion and a mild mixing necessary to account for the lithium depletion. Then the comparison between the models and the "seismic sun" is very good below the convection zone. However in this model the ${ }^{3} \mathrm{He}$ increase with time is too large compared to the observations.

More recent solar models have been computed with a smaller mildly mixed zone below the convection zone in order to account for the lithium depletion and the constraint on the ${ }^{3} \mathrm{He}$ enhancement (Vauclair and Richard 1997a). In these models the critical $\mu$-gradient able to stabilize the mixing processes was varied as a parameter, with $(\nabla \ln \mu)_{c r i t}$ between 5 and $1.5 \times 10^{-13} \mathrm{~cm}^{-} 1$. The variation of the ${ }^{3} \mathrm{He} /{ }^{4} \mathrm{He}$ ratio was found small enough during these last 3 Gyrs as soon as $(\nabla \ln \mu)_{\text {crit }} \leq 2 \times 10^{-13}$.

If the constraint on the beryllium depletion is relaxed, it allows a still thinner mixed zone. It is interesting in this case to compute the minimum enhancement of the ${ }^{3} \mathrm{He} /{ }^{4} \mathrm{He}$ implied by the lithium observed depletion. Vauclair and Richard (1997b) show that it is possible to deplete lithium by a factor larger than 100 as observed and not increase ${ }^{3} \mathrm{He} /{ }^{4} \mathrm{He}$ by more than 5 percent since the solar origin. In this case beryllium is only depleted by about 10 percent.

\section{Helioseismology and the Solar Neutrino Problem}

The comparison between the computed solar models and the "seismic Sun" (Figures 1 and 2) is also able to rule out the core mixing processes which have been invoked to account for the solar neutrino deficiency (e.g. Morel and Schatzman 1996, Cumming and Haxton 1996). Such a mixing, which could be induced by internal waves, nuclear instabilities or any other process, remains an a priori possibility to decrease the solar neutrino flux. The basic reason is that it brings ${ }^{3} \mathrm{He}$ down towards the solar center and increases the rate of the ${ }^{3} \mathrm{He}\left({ }^{3} \mathrm{He}, 2 \mathrm{p}\right){ }^{4} \mathrm{He}$ nuclear reaction yield, while the ${ }^{3} \mathrm{He}$ $\left({ }^{4} \mathrm{He}, \alpha\right){ }^{7} \mathrm{Be}$ reaction is reduced.

In the present computations (Richard and Vauclair 1997), we have introduced in the best solar model of RVCD a parametrized mixing region located at the edge of the nuclear burning core. We have introduced this extra-mixing in the form of a gaussian centered at a radius $\mathrm{r} / \mathrm{R}_{\odot}=.2$, with a maximum turbulent diffusion coefficient of 1000 $\mathrm{cm}^{2} . \mathrm{s}^{-1}$, and a width $\delta / \mathrm{R}_{\odot}=.04$, similar to the parametrization introduced by Morel and Schatzman (1996).

Such a core mixing can indeed reduce the neutrino fluxes. Table 1 presents the luminosity, radius, and neutrino production for the two models (RVCD : unmixed in the core; cmix : core mixed). The neutrino fluxes are smaller in the second model, close to the detection values. However this solar model is inconsistent with helioseismology (Figure 1). 


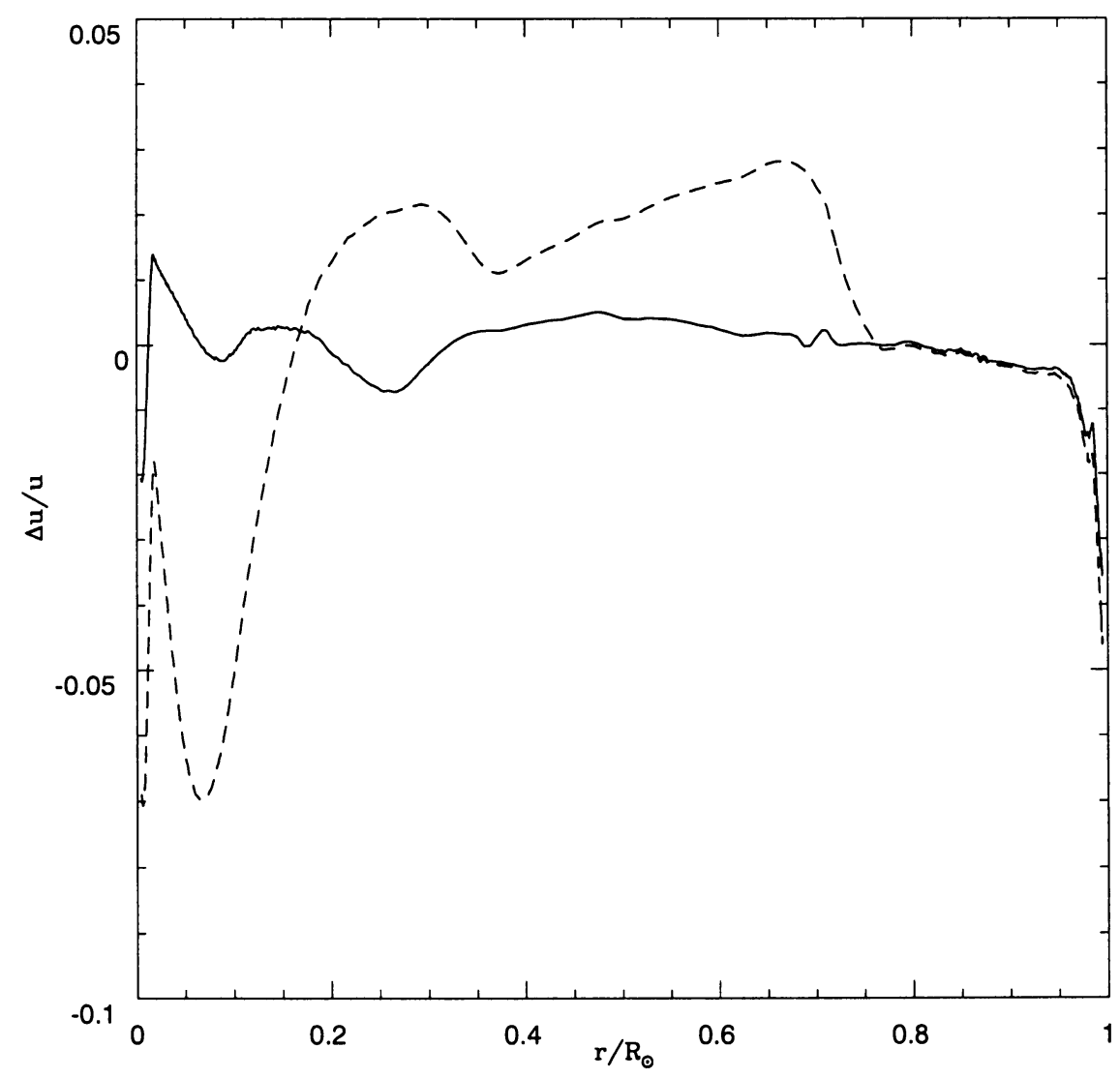

Figure 1. Differences between the $u=P / \rho$ function in the seismic Sun and the best model of RVCD (solid line), and a model including core mixing (dashed line). Mixing near the solar core leads to a modification of the solar structure which is incompatible with the seismic Sun (after Vauclair and Richard 1997a).

\section{Conclusion}

In summary, the constraints implied by both the helioseismic inversions and abundance determinations in the Sun converge towards the existence of a small mild mixing region below the convection zone, which would extend down to a depth of the order of one scale height. The implied mixing region must be very mild, with diffusion coefficients of $10^{3}-10^{4}$ only. This is quite different from a traditional adiabatically stratified overshooting zone, which is excluded from helioseismology (Christensen-Dalsgaard et al 1995, Basu 1997). It could be related to the differential rotation which occurs below the convection zone.

Such a mild mixing zone can lead to a lithium depletion by a factor 140 as observed, without increasing too much the ${ }^{3} \mathrm{He} /{ }^{4} \mathrm{He}$ ratio. In this case beryllium is not 

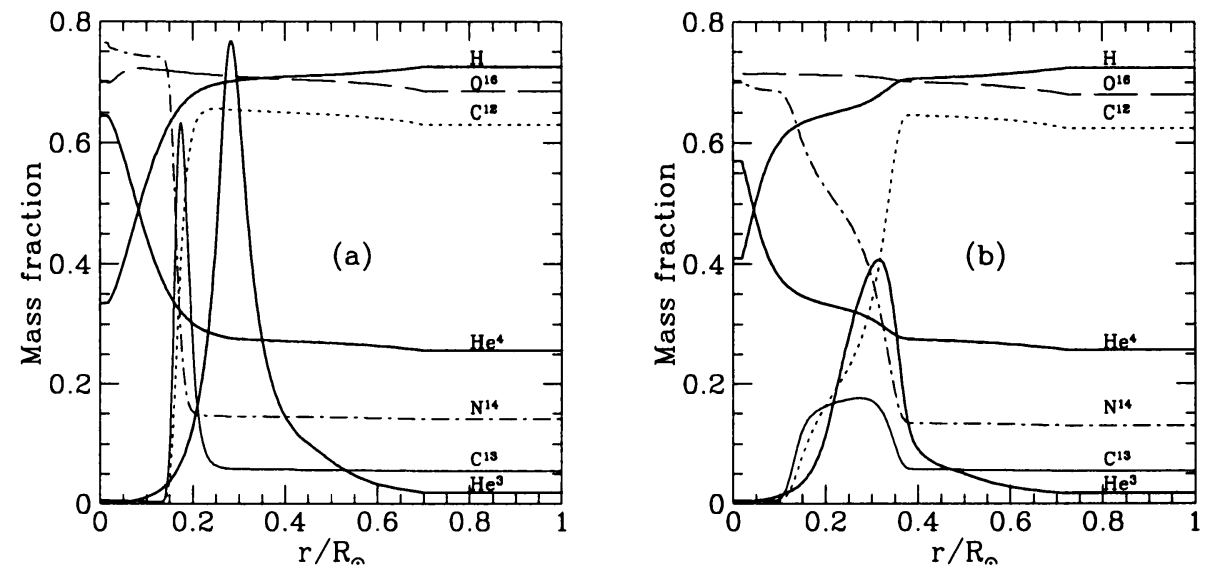

Figure 2. Chemical composition inside solar models (the conventions are the same as in RVCD). Graph (a) represents the case of the best solar model in Richard et al., with no core mixing. The influence of the mixing layer below the convection zone on the ${ }^{3} \mathrm{He}$ abundance is clearly seen on the right side of the ${ }^{3} \mathrm{He}$ peak. Graph (b) represents the model with a partially mixed core. In this model the neutrino fluxes are decreased, but it is not compatible with helioseismology.

TABLE 1.

\begin{tabular}{cccccc}
\hline model & $\begin{array}{c}\mathrm{L} \\
\left(10^{34} \mathrm{erg} \cdot \mathrm{s}^{-1}\right)\end{array}$ & $\begin{array}{c}\mathrm{R} \\
\left(10^{11} \mathrm{~cm}\right)\end{array}$ & $\begin{array}{c}\phi\left({ }^{8} \mathrm{~B}\right) \\
\left(10^{6} \mathrm{~cm}^{2} \mathrm{~s}^{-1}\right)\end{array}$ & $\begin{array}{c}(\phi \sigma) \mathrm{Cl} \\
\left(\mathrm{SNU}_{S}\right)\end{array}$ & $\begin{array}{c}(\phi \sigma) \mathrm{Ga} \\
\left(\mathrm{SNU}_{S}\right)\end{array}$ \\
\hline RVCD & 0.3851 & 0.6960 & 6.06 & 8.14 & 130.84 \\
cmix & 0.3857 & 0.6959 & 2.60 & 3.90 & 108.75 \\
Observed & $0.3851 \pm 0.0005$ & $0.6959 \pm 0.0001$ & $2.80 \pm 0.19 \pm 0.33$ & $2.54 \pm 0.14 \pm 0.14$ & $\begin{array}{c}\text { Sage: } 72_{-10-7}^{+12+5} \\
\end{array}$ \\
& & & & & Gallex: $69.7 \pm 6.7_{-4.5}^{+3.9}$ \\
\hline
\end{tabular}

depleted, which is consistent with Balachandran (1997) suggestion. The computed sound velocity is very close to that derived from inversion procedures. The helium profiles directly obtained from helioseismology (Basu 1997, Antia and Chitre 1997) show indeed a helium gradient below the convection zone which is smoother than the gradient computed from pure element segregation. On the other hand, this gradient is compatible with the one obtained when the mild mixing region is added.

In any case this localized mixing region must be completely disconnected from the solar core. The $\mu$-gradient may play the role of a cut-off in this respect. No mixing can indeed be allowed in the nuclear energy production region as it would lead to a sound velocity incompatible with helioseismology. In particular the mixing processes invoked to decrease the neutrino fluxes are excluded. 


\section{References}

Antia, H.M., Chitre, S.M..: 1997, submitted to A\&A

Bahcall, J.N., Pinsonneault, M.H.: 1992, Reviews of Modern Physics 64, 885

Balachandran, S.: 1997, preprint

Basu, S.: 1997, Mon. Not. R. astr. Soc., 288, 572

Charbonnel, C., Vauclair, S., Zahn, J.P.: 1992, A\&A 255, 191 (CVZ)

Cumming, A., Haxton, W.C. : 1996 Phys. Rev. Lett. 77, 4286

Christensen-Dalsgaard, J., Monteiro, M.J.P.F.G., Thompson, M.J.: 1995, MNRAS 276, 283

Christensen-Dalsgaard, J., Proffitt, C.R., Thompson, M.J.: 1993, ApJ 408, L75

Cox, A.N., Guzik, J.A., Kidman, R.B.: 1989, ApJ 342, 1187

Dziembowski, W.A., Goode, P.R., Pamyatnikh, A.A., Sienkiewicz, R.: 1994, ApJ 432, 417

Geiss, J.: 1993, Origin and Evolution of the Elements, ed. Prantzos, Vangioni-Flam \& Cassé (Cambridge Univ. Press), 90

Gloecker, G., Geiss, J.: 1997 preprint

Grevesse, N.: $1991, A \& A$ 242, 488

Michaud, G.: 1970, $A p J$ 160, 641

Morel, P., Schatzman, E.: 1996, $A \& A$ 310, 982

Paquette, C., Pelletier, C., Fontaine, G., Michaud, G.: 1986, ApJS61, 177

Proffitt, C.R.: 1994, ApJ 425, 849

Richard, O., Vauclair, S., Charbonnel, C., Dziembowski, W.A.: 1996, $A \& A$ 312, 1000

Richard, O., Vauclair, S.: $1997, A \& A$ 322, 671

Schatzman, E.: 1945, Ann. d'Astr. 8, 143

Thoul, A.A., Bahcall, J.N., Loeb, A.: 1994, ApJ 421, 828

Vauclair, S., Vauclair, G.: 1982, ARA\&A 20, 37

Vauclair, S., Richard, O.: 1997a, Proceedings of the Los Alamos meeting "a half century of stellar pulsation interpretations : a tribute to Arthur N. Cox", June 16-20 , 1997

Vauclair, S., Richard, O.: 1997b, preprint 